\title{
Semileptonic and missing energy results from early Belle II data
}

\section{William Sutcliffe ${ }^{* \dagger}$}

Karlsruhe Institute of Technology

E-mail: william.sutcliffe2@kit.edu

\begin{abstract}
Semileptonic and missing energy decays form a large part of the Belle II physics program from determinations of the CKM matrix elements $\left|V_{u b}\right|$ and $\left|V_{c b}\right|$ to measurements of observables that are sensitive to physics such as $R_{D^{*}}$ and $\mathcal{B}\left(B \rightarrow K^{*} v \bar{v}\right)$. In these proceedings first reconstructions of semileptonic decays $B^{0} \rightarrow D^{*+} \ell v$ and $B \rightarrow X \ell v$ in $5.15 \mathrm{fb}^{-1}$ of Belle II data are presented with and without hadronic tag-side reconstruction.
\end{abstract}

European Physical Society Conference on High Energy Physics - EPS-HEP2019 -

10-17 July, 2019

Ghent, Belgium

* Speaker.

${ }^{\dagger}$ On behalf of the Belle II collaboration 


\section{Introduction}

The Belle II experiment [1] is an $e^{+} e^{-}$collider experiment in Japan, which began its main physics run in early 2019 and has collected $5.15 \mathrm{fb}^{-1}$ of data at a centre-of-mass energy corresponding to the mass of the $\Upsilon(4 S)$ resonance. The clean environment of $e^{+} e^{-}$collisions together with the unique event topology of Belle II, in which an $\Upsilon(4 S)$ meson is produced and subsequently decays in to a pair of $B$ mesons, allows a wide range of physics measurements to be performed which are difficult or impossible at hadron colliders. In particular, measurements in which there is missing energy, which includes semileptonic decays with missing neutrinos, can benefit substantially from the additional constraints provided by the collision environment of Belle II.

Measurements of semileptonic decays of $B$ mesons are essential for precision determinations of elements of the CKM matrix. These measurements in turn can be used in global fits to determine the four parameters of the Standard Model (SM) in the CKM sector. Belle II will provide a key role in improving the precision on determinations of the CKM matrix elements $\left|V_{u b}\right|$ and $\left|V_{c b}\right|$ using $b \rightarrow u \ell v$ and $b \rightarrow c \ell v$ transitions. In addition, measurements of semileptonic decays and decays with missing energy are excellent probes of new physics and could potentially resolving several anomalies from the SM observed in $B$ physics $[2,3]$. This includes the $3 \sigma$ tension seen in the ratio of $R\left(D^{(*)}\right)=\mathcal{B}\left(B \rightarrow D^{(*)} \tau v\right) / \mathcal{B}\left(B \rightarrow D^{(*)} l v\right)_{\ell=e, \mu}$, where Belle II will have a large impact. Additionally, Belle II will be able to measure $b \rightarrow s v \bar{v}$, which will provide an orthogonal probe to the measurements of $b \rightarrow s \ell^{+} \ell^{-}$, where several discrepancies with the SM have been observed.

In these proceedings first reconstructions of semileptonic decays in early Belle II data (5.15 $\mathrm{fb}^{-1}$ ) are presented using two complementary reconstruction techniques either with or without tag-side reconstruction.

\section{Semileptonic decays with hadronic tag-side reconstruction}

\subsection{Hadronic tag-side reconstruction at Belle II}

A critical technique for many of these measurements is tag-side $B$ meson reconstruction, in which the non-signal $B$ meson $\left(B_{\mathrm{tag}}\right)$ in the event is reconstructed. This is illustrated in Figure 1(a), in which the decay $B^{+} \rightarrow X^{0} \ell^{+} v_{l}$ is reconstructed as signal. By reconstructing the tag-side important constraints on the flavour and kinematics of the signal $B$ can be obtained. The tag-side in Figure 1 is reconstructed in a given exclusive final state $B_{\text {tag }}^{+} \rightarrow\left(D^{0} \rightarrow K^{-} \pi^{+}\right) \pi^{+}$, which is known as exclusive tag-side reconstruction. In practice a large number of exclusive modes are reconstructed in order to cover a larger branching fraction of the $B$ meson.

The Belle II tag-side reconstruction algorithm, which improves over its Belle predecessor Full Reconstruction [4], is known as Full Event Interpretation [5]. This algorithm performs a hierarchical reconstruction of $B$ meson decay chains utilising multivariate classification at each stage. In particular the algorithm. starts from final state particles such as electrons, muons, kaons, pions and photons and employs several levels of reconstruction of intermediate particles $\left(D, D^{*}, K_{s}^{0}\right.$, $J / \psi$ and $\pi^{0}$ ), which belong to the $B$ meson decay, before finally reconstructing the $B$ meson decay. For each final state particle or unique decay of a none stable particle a boosted decision tree is trained to classify whether or not the particles are correctly reconstructed. The algorithm considers $\mathcal{O}(200)$ decay channels and, thereby, reconstructs $\mathcal{O}(10000)$ unique $B$ meson decay chains. 
(a)

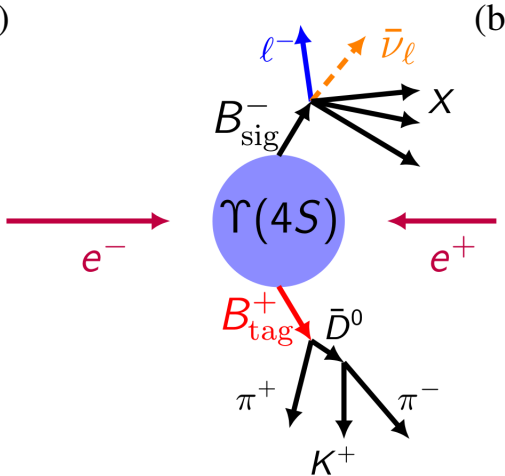

(b)

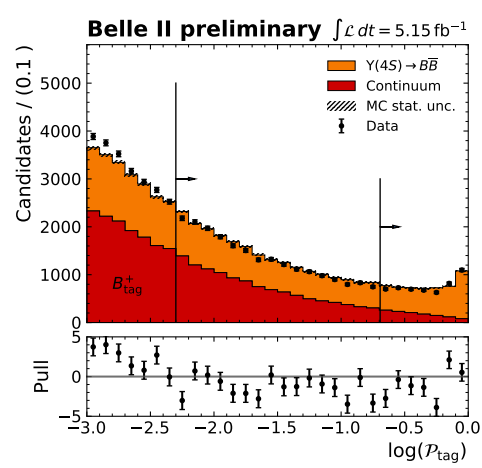

Figure 1: (a) Diagram illustrating the use of tag-side reconstruction to search for the decay $B^{+} \rightarrow X^{0} \ell^{+} v_{l}$. The tag-side $B$ meson, $B_{\text {tag }}$ is reconstructed hadronically as $B_{\mathrm{tag}}^{+} \rightarrow\left(D^{0} \rightarrow K^{-} \pi^{+}\right) \pi^{+}$. Knowledge of the tagside flavour and 4-momentum imposes constraints on the flavour and kinematics of the signal-side meson. (b) Comparison of the distribution of $\log \mathcal{P}_{\text {tag }}$ in early Belle II data to the shape expectation from simulation. Here $\log \mathcal{P}_{\text {tag }}$ is the logarithm of the tag-side $B^{+}$meson classifier output, $\mathcal{P}_{\text {tag }}$. Reference selection criteria of $\mathcal{P}_{\text {tag }}>0.1$ and $\mathcal{P}_{\text {tag }}>0.5$ are illustrated.
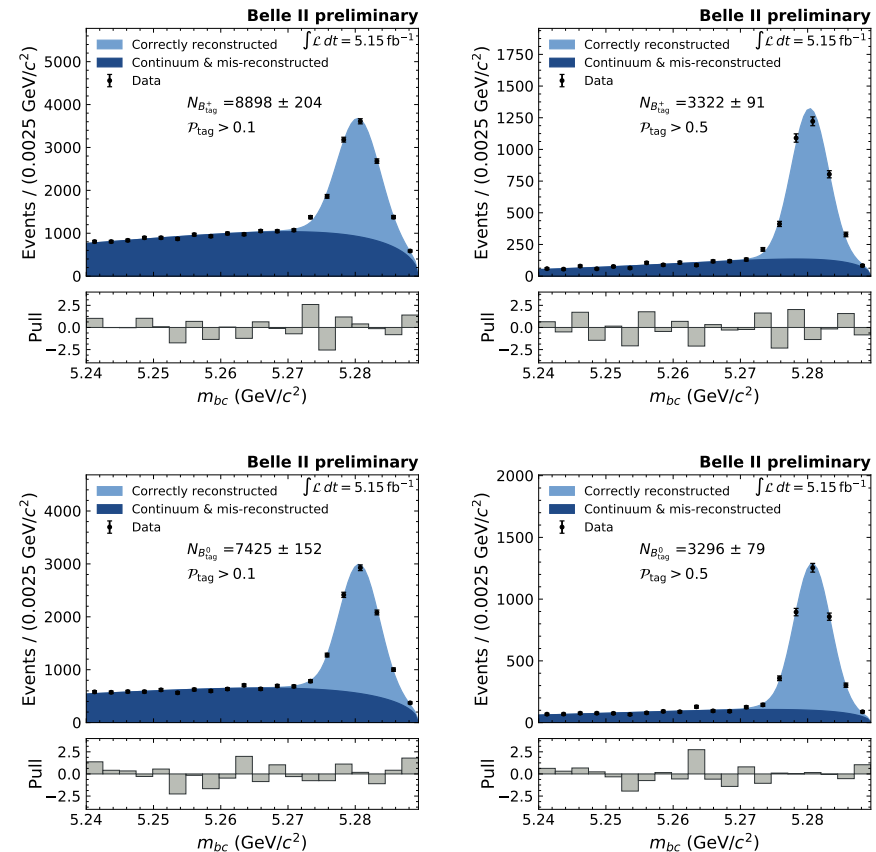

Figure 2: Fits to the beam constrained mass, $m_{b c}$, distribution of reconstructed $B^{+}$(top) and $B^{0}$ (bottom) tag-side $B$ mesons in data. A looser selection criteria of $\mathcal{P}_{\text {tag }}>0.1$ (left) and a tighter selection criteria of $\mathcal{P}_{\text {tag }}>0.5$ are applied on the $B$ meson classifier $\mathcal{P}_{\text {tag }}$ to select samples with different levels of purity.

The final multivariate classifier output, $\mathcal{P}_{\text {tag }}$ of the reconstructed $B$ meson can be used to select a pure sample of correctly reconstructed $B$ mesons. Figure 1(b) shows a comparison of the shape of the $\log \mathcal{P}_{\text {tag }}$ distribution in data with that from simulation. Here tag-side $B$ mesons are required to 
have a beam energy difference, $\Delta E=E_{\text {tag }}^{*}-E_{\text {beam }}{ }^{1}$ between -0.15 and $0.1 \mathrm{GeV} / \mathrm{c}$, in addition to, loose selections on an event level normalised 2nd fox wolfram moment and the cosine of the thrust axis of the $B$ meson to that of the other particles in the event are applied to remove background from $e^{+} e^{-} \rightarrow q \bar{q}$. Finally, only the candidate with the highest classifier value, $\mathcal{P}_{\text {tag }}$ is kept in each event. Correctly reconstructed tag-sides peak towards a classifier values of $\mathcal{P}_{\text {tag }}=1$ resulting in the peak observed at $\log \left(\mathcal{P}_{\text {tag }}\right)=0$. A better agreement between simulation and data is observed at higher values of $\log \mathcal{P}_{\text {tag }}$ because here the distribution is dominated by higher purity modes, which are easier to reconstruct and often better modelled in the simulation.

The performance of tag-side reconstruction will have a large impact on a varitey of the measurements, for which its use is essential. In particular, the tag-side efficiency, which is the number of correctly reconstructed tag-sides for a given number of $\Upsilon(4 S)$ collision events, will factorise into the overall signal efficiency of an analysis. In addition, the purity, which is the percentage of events with a tag-side which have a correct tag-side, will influence the quality of signal reconstruction and background levels in the final analysis. It is possible to select a sample with a higher purity by applying a selection on $\mathcal{P}_{\text {tag }}$. This is demonstrated in Figure 2, which shows fits to the beam constrined mass distribution, $m_{b c}=\sqrt{E_{\text {beam }}^{2}-p_{\text {tag }}^{* 2}}$, for reconstructed tag-side $B^{0}$ and $B^{+}$mesons, for selections of $\mathcal{P}_{\text {tag }}$ greater than 0.1 and 0.5 . The contribution from correctly reconstructed tag-side $B$ mesons is parametrised by a crystal balli, meanwhile, background from $e^{+} e^{-} \rightarrow q \bar{q}(q=u, d, s, c)$ and incorrectly reconstructed $B$ mesons is modelled with an argus function. By applying a tighter selection on the classifier output a higher purity sample of tag-side $B$ mesons can be selected with the sacrifice of a lower tag-side efficiency, which is proportional to the yield of correctly reconstructed tag-side $B$ mesons.

\section{2 $B \rightarrow X \ell v$ decays with hadronic tag-side reconstruction}

Given the large number of multivariate classifiers employed during tag-side reconstruction there can be differences between the tag-side reconstruction efficiency in simulation and data. In order, to correct for this a calibration can be performed by measuring a decay with a well known branching fraction and sufficient available statistics after selection. A suitable choice given the current Belle II dataset is inclusive $B \rightarrow X \ell v$ decays because of the substantial branching fraction of $\sim 20 \%$. This is also an ideal choice of decay to demonstrate the applicability of tag-side reconstruction to inclusive semileptonic decays in Belle II data.

In order to have one tag-side in each event a best candidate selection was made on $\mathcal{P}_{\text {tag }}$. Subsequently the tag-side is required to have $\mathcal{P}_{\text {tag }}$ greater than 0.01 and a $m_{b c}$ value within the signal window for correctly reconstructed signal $\left(m_{b c}>5.27 \mathrm{GeV} / \mathrm{c}^{2}\right)$. After selecting a suitable tag-side additional signal side selection can be applied. In particular, a lepton is selected with $p_{\ell}^{*}>0.6 \mathrm{GeV} / \mathrm{c}$ and basic impact parameter cuts. Particle identification information from several sub-detectors, including Cherenkov time of progation (TOP), Aerogel ring imaging Cherenkov and dedicated muon detectors, is combined into a likelihood for each of electron and muon hypotheses in order to select each lepton species.

Backgrounds can be divided into three categories: $e^{+} e^{-} \rightarrow q \bar{q}$, cases in which the lepton is a mis-identified hadron (fake $\ell$ ) and $\Upsilon(4 S) \rightarrow B \bar{B}$ decays where the lepton is not produced directly

\footnotetext{
$1 *$ here refers to the centre-of-mass frame.
} 

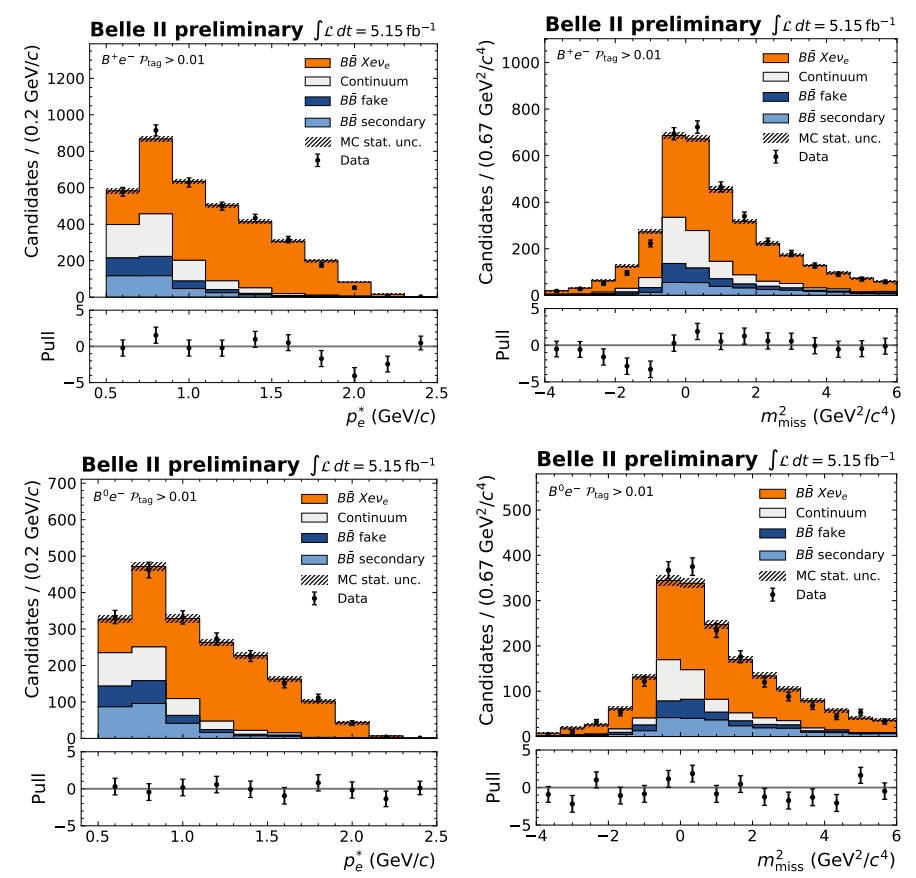

Figure 3: Comparison of the centre-of-mass momentum, $p_{e}^{*}$, (left) and missing mass squared, $m_{\text {miss }}^{2}$, (right) distributions in data to the shape expected from Monte Carlo simulation for $B_{\mathrm{tag}}^{+} e^{-}$(top) and $B_{\mathrm{tag}}^{0} e^{-}$(bottom) combinations.

from a decay of a $B$ meson (secondary $l$ ). The semileptonic $B \rightarrow X l v$ signal can be separated from background by either performing a fit to the centre-of-mass momentum distribution, $p_{\ell}^{*}$, or a missing mass squared, $m_{\text {miss }}^{2}$ variable. Here, the variable, $m_{\text {miss }}^{2}$, is determined by using tracks and electromagnetic clusters in the rest of the event to reconstruct the 4-momentum of $X, p_{X}$. Subsequently, the missing mass squared, $m_{\text {miss }}^{2}$, is computed as,

$$
m_{\mathrm{miss}}^{2}=\left(p_{e^{+} e^{-}}^{*}-p_{B_{\mathrm{tag}}}^{*}-p_{\ell}^{*}-p_{X}^{*}\right)^{2} .
$$

Figure 3 shows a comparison of the observed shape of the $p_{\ell}^{*}$ and $m_{\text {miss }}^{2}$ distributions in data with the expectation from simulation for inclusive $B^{0} \rightarrow X^{+} e^{-} v_{e}$ and $B^{-} \rightarrow X^{0} e^{-} v_{e}$ decays. A clear peaking contribution is seen at higher $p_{\ell}^{*}$ from semileptonic $B \rightarrow X e v_{e}$ decays in $p_{e}^{*}$. There appears to be some disagreement in the $p_{e}^{*}$ spectrum for $B^{-} \rightarrow X^{0} e^{-} v_{e}$ decays. This is most likely due to the fact that corrections to update the composition and form factors of semileptonic $B \rightarrow X_{c} \ell v$ modes have not been applied.

The results in this section have demonstrated the applicability of tag-side reconstruction in early Belle II data, which will be critical for a number of analyses. Furthermore, it is already possible to reconstruct a sample with $\mathcal{O}(1000) B \rightarrow X \ell v_{l}$ decays, which will be sufficient to calibrate the algorithm in early data.

\section{Semileptonic decay reconstruction without tag-side reconstruction}

Although hadronic tag-side reconstruction can provide essential constraints for the reconstruc- 
tion of challenging signal sides, the efficiency incurred in the reconstruction of the tag-side will result in a sacrifice in the potential signal-side statistics of an analysis. In addition, hadronic tag-side reconstruction will also contribute a systematic efficiency associated with calibrating its efficiency mismatch between data and simulation. For these reasons it can be often advantageous and complementary to perform an analysis without tag-side reconstruction (untagged).

Here an untagged study of $B^{0} \rightarrow\left(D^{*} \rightarrow \pi^{+}\left(D^{0} \rightarrow K^{+} \pi^{-}\right)\right) \ell^{-} v$ in early Belle II data is presented. This provides an important benchmark to demonstrate the possibility to study semileptonic decays effectively at Belle II. In addition, this decay is important as it can be used to determine the CKM matrix element $\left|V_{c b}\right|$.
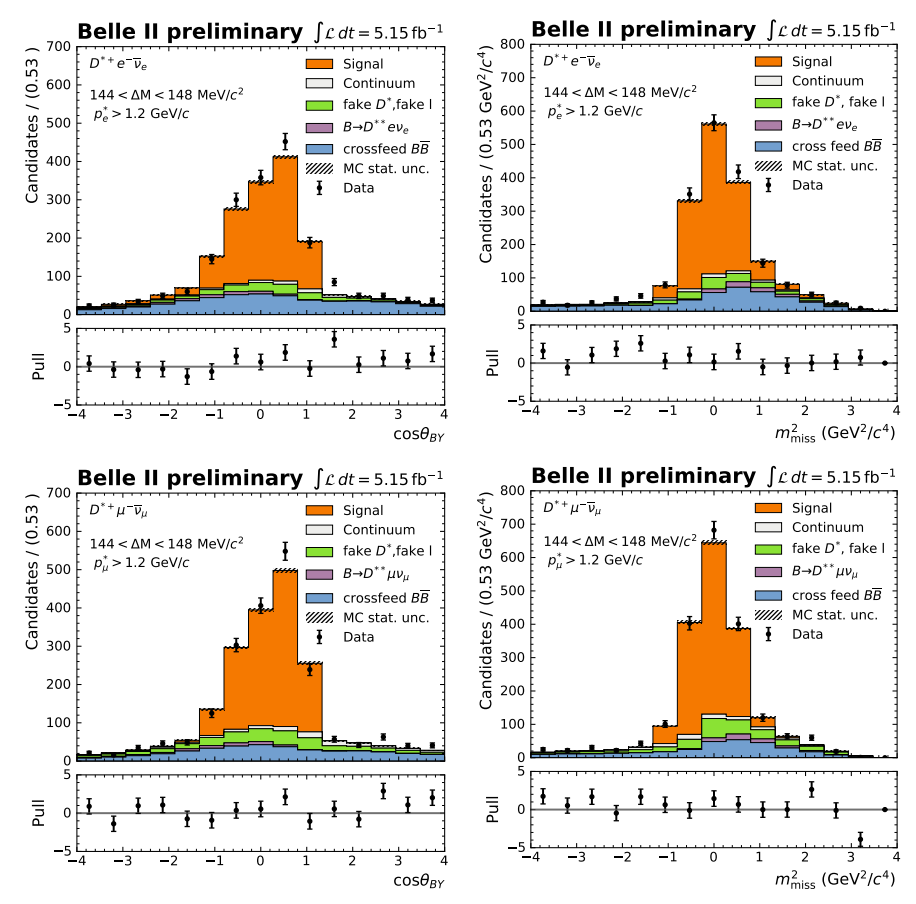

Figure 4: Fits to the $\cos \theta_{B D^{*} \ell}$ and $m_{\text {miss }}^{2}$ distributions for reconstructed $B^{0} \rightarrow\left(D^{*} \rightarrow \pi^{+}\left(D^{0} \rightarrow K^{+} \pi^{-}\right)\right) \ell^{-} v$ decays.

The selection for $B^{0} \rightarrow\left(D^{+*} \rightarrow \pi^{+}\left(D^{0} \rightarrow K^{+} \pi^{-}\right)\right) \ell^{-} v$ decays demands a high momentum lepton, $p_{\ell}^{*}>1.2 \mathrm{GeV} / \mathrm{c}$, with a similar particle identification selection as mentioned in the previous section for $B \rightarrow X \ell v$ decays. All tracks are required to come from near the interaction point by requiring that the impact parameter in $z$ direction is less than $2 \mathrm{~cm}$ and $0.5 \mathrm{~cm}$ in the $r-\phi$ plane. In addition, the kinematic endpoint of the momentum distribution of the charged pion, $\pi^{+}$, from the $D^{*}$ decay is exploited by requiring it to have momentum less than $0.5 \mathrm{GeV} / \mathrm{c}$. While no PID requirements are placed on the pions and kaons in the decay, mass selections are made on the $D^{0}$ mass, $1.85<m_{D^{0}}<1.88 \mathrm{GeV} / \mathrm{c}^{2}$ and the mass difference, $\Delta m$, between the $D^{*}$ and the $D$ meson, $0.144<\Delta M<0.148 \mathrm{GeV} / c^{2}$. The momentum of the $D^{*}$ is required to be less than $2.5 \mathrm{GeV} / \mathrm{c}$, which removes background $D^{*}$ mesons from $e^{+} e^{-} \rightarrow c \bar{c}$ events while retaining signal. Finally, $e^{+} e^{-} \rightarrow q \bar{q}$ is further suppressed by requiring that the normalised second order Fox Wolfram Moment is less than 0.25 . 
The determination of the signal contribution is determined using fits to $\cos \theta_{B D^{*} \ell}$ and $m_{\text {miss }}^{2}$. Where here, $\cos \theta_{B D^{*} \ell}$ is the angle between the $B$ meson and $D^{*} \ell$ momenta in the centre-of-mass frame, which is constrained to physical values between -1 and 1 for correctly reconstructed $B \rightarrow$ $D^{*} \ell v$ decays. Meanwhile, $m_{\text {miss }}^{2}$, is the missing mass squared, which has been computed in the centre-of-mass frame according to the following approximation:

$$
m_{\text {miss }}^{2}=\left(\left(E_{\text {beam }} / 2,0,0,0\right)-p_{D^{*} \ell}^{*}\right)^{2},
$$

which assumes that the both $B$ mesons are at rest in this frame.

Figure 4 shows independent fits to the $\cos \theta_{B D^{*} \ell}$ and $m_{\text {miss }}^{2}$ distributions for both electron and muon channels. Backgrounds from $D^{* *} \ell v$ decays, mis-identified hadrons as leptons (fake $\ell$ ), $e^{+} e^{-} \rightarrow q \bar{q}$ and combinatorial cross-feed are considered. Yields consistent within $2 \sigma$ from one another were observed between the fitting approaches.

\section{Conclusion and outlook}

In these proceedings first reconstructions of semileptonic decays with the full Belle II detector in place were presented, which utilise $5.15 \mathrm{fb}^{-1}$ of data. Two complementary approaches of reconstruction were employed. The first of which relies on tag-side reconstruction, in which, one $B$ meson in the event is reconstructed. Meanwhile, the second of which utilises only information from the semileptonic decay itself and is applied here to reconstruct $B^{0} \rightarrow\left(D^{*} \rightarrow \pi^{+}\left(D^{0} \rightarrow K^{+} \pi^{-}\right)\right) \ell^{-} v$.

First results from the Belle II tag-side reconstruction algorithm, Full Event Interpretation, presented here demonstrate that the algorithm is performing well in early data. In addition, a first signal-side reconstruction of $B \rightarrow X \ell v$ decays was made with the aim of ultimately calibrating the algorithm. Tag-side reconstruction will be critical to measure decays with missing energy such as $B \rightarrow s v \bar{v}, B \rightarrow D^{*}(\tau \rightarrow \ell v \bar{v}) \nu$ and inclusive semileptonic decays, $B \rightarrow X l v$. In particular, it is expected that the uncertainty on $R_{D^{*}}$ with $5 \mathrm{ab}^{-1}\left(50 \mathrm{ab}^{-1}\right)$ utilising tag-side reconstruction will be 3\% (2\%) [1]. Meanwhile, it will be possible to discover $B \rightarrow K^{*} v \bar{v}$ with an uncertainty of $25-30 \%(10 \%)$ [1]. A complementary approach is to study semileptonic decays without tag-side reconstruction. Here such a study was performed for $B^{0} \rightarrow\left(D^{*} \rightarrow \pi^{+}\left(D^{0} \rightarrow K^{+} \pi^{-}\right)\right) \ell^{-} v$ decays. In the future the Belle II experiment, with its ultimate dataset, will be able determine $\left|V_{u b}\right|$ and $\left|V_{c b}\right|$ with uncertainties of $\sim 1 \%$, which will have a substantial impact on global fits in the CKM sector [1].

Together reconstructions of $B^{0} \rightarrow\left(D^{*} \rightarrow \pi^{+}\left(D^{0} \rightarrow K^{+} \pi^{-}\right)\right) \ell^{-} v$ untagged and $B \rightarrow X \ell v$ tagged are important first milestones in the Belle II physics program.

\section{References}

[1] Altmannshofer W et al, The Belle II physics book, (2018) [arXiv:1808.10567]

[2] G. Ciezarek et al, Nature 546 (2017) 227-233

[3] F. Archilli et al, Nature 546 (2017) 221-226

[4] Feindt M et al, Nucl. Instrum. Meth. $\mathbf{A 6 5 4}$ (2011) 432-440

[5] Keck T et al Comput Softw Big Sci 3 (2019) 6 